No. 11.

AUX ELECTEURS

\title{
LE GOUVERNEMENT \\ ET \\ LES CULTIVATEURS
}

\section{FERMES EXPERIMENTALES}

La ferme expérimentale centrale à Ottawa, a été établie en 1856, et immédiatement après, des succursales furent créées d̀ Nappan, N. E., Brandon, Man., Indian Head, T. N. O., et à Agassiz, C. A.

Ce système, placé sous la direction active et, intelligente du ministre de l'agriculture, est l'un des plus complets du monde entier. Il peut être de la plus grande utilité aux cultivateurs du Canada.

Le fait que cette institution est appréciée par la classe qu'elle est destinée à aider, est prouvé par la demande de nourelles variétés de grains de semence, par les renseignements demandés, et par la circulation croissante des bulletins. Ces bulletins font connaître les résultats obtenus de temps à autre, et ils sont préparés avec le plus grand soin sous le rapport de l'exactitude des détails et de la clarté. On les considère comme étant dignes de la plus grande confiance. 
Il a été expédié l'an dernier de dix-huit à dix-neuf tonnes d'échantillons de grains. La liste de ceux qui recoivent les bulletins contient les noms de 20,600 cultivateurs résidant dans toutes les parties du Canada, et dans le cours de l'année 36,362 lettres ont été échangées entre des particuliers et les employés de la ferme.

En 1890, l'on a fait des essais à la ferme centrale avec 1,240 échantillons de grains de semence, dans le but de renseigner les cultivateurs sur ceux qui pourraient être cultivés dans des conditions favorables. Ces reuseignements sont fournis gratuitement aux cultivateurs.

Les échantillons.leur sont expédiés par la malle, franc de port, et ils n'ont rien à payer pour l'épreuve qui en a été faite.

De plus, les employés sont toujours prêts à répondre promptement et gratuitement à toutes demandes de renseignements. Ils feront même plus que cela sans rien exiger. Ainsi, par exemple, le chimiste recevra de la part des cultivateurs des échantillons d'eau de puits, et en temps voulu il leur dira si elle est pure et propre à l'usage, soit pour eux ou leurs animaux. Si un cultivateur trouve un insecte ravageant ses fruits, ses légumes ou son grain, il n'a qu'à en expédier un spécimen à l'entomologiste, et il recevra une réponse précisant la nature de l'insecte et le remède à employer.

L'horticulteur résout les problèmes se rapportant ì la culture des fruits ou des arbres, et les questions qui se rapportent à l'élevage des volailles sont traitées par un expert habile qui contrôle ce département.

En outre, le directeur est prêt à recevoir toute demande de renseignements relativement à la culture, et à répondre chaque fois que la chose est possible. Ces fermes sont complètement et parfaitement équipées, et elles sont destinées à augmenter les bénéfices de la culture dans toutes ses branches.

\section{L'orge à deux rangs}

En 1889 nous arons eu une preure que le gourernement fitit tout en son pouvoir pour aider les cultivateurs. Pour des motifs qui sont généralement bien compris, le ministre de l'agriculture a inporté d'Angleterre 10,000 boisseaux de la plus belle orge propre 
au maltage. Cette orge a été vendue aux cultivateurs de tout le Canada au prix conttant en Angleterre. L'année a été défavorable ì la culture de l'orge, mais la récolte de la dernière saison, provenant de l'orge importée, a bien réussi. Des échantillons ont été expédiés l'autonme dernier, à l'exposition des brasseurs et des malteurs, à Londres, et ils ont été fort appréciés.

Tout dernièrement, 400 boisscaux d'orge ont été expédiés en Angleterre dans le but d'en faire faire l'essai dans une des principales brasseries. Les rapports des experts, dans le Royaume-Uni, sans une seule exception, prouvent que l'orge à deux rangs peut se cultiver en Canada avec succès, et être de la plus belle qualité, telle que celle qui a obtenu un prix élevé en Angleterre. En conséquence, si le marché pour leur orge est fermé aux Etats-Unis, nos cultivateurs peuvent en trouver un en Angleterre-pourvu, naturellement, qu'ils cultivent celle qui a la qualité requise et qu'ils lui donnent le soin et l'attention nécessaires.

\section{Commissaire de l'industrie laitière}

La nomination du professeur J. W. Robertson à la charge de commissaire de l'industrie laitière au Canada, a été une autre preuve frappante que le gouvernement sait apprécier les besoins de nos cultivateurs. Le développement d'un commerce considérable de fromage et l'accroissement de la production des produits de la laiterie dans tout le Canada, exigeaient tout l'aide que le gouvernement pouvait accorder à cette industrie.

Les laitier's du pays, réunis en convention, suggérèrent la nomination d'un commissaire, et le ministre de l'agriculture y consentit immédiatement. La nomination du professeur Robertson a été farurablement accueillie par tous les laitiers du Canada, et il a déjà su rendre de grands services. Il fait partie du personnel de la ferme expérimentale centrale, et il a pris la direction d'une laiterie magnifiquement organisée.

\section{Ecoles d'industrie laitière}

Le gouvernement a fait des arrangements pour établir des ecoles d'industrie laitière daus le but louable d'enseigner aux cul- 
tivateurs la manière de bien appliquer leur habileté et leur travail a la production du beurre et du fromage de belle qualité. Ces écoles seront sous le contrôle du commissaire de l'industrie laitière. On sait que le Danemark ne possédant pas de facilités naturelles meillenres que celles que nous avons en Canada, a donné à ses produits de la laiterie une plàce proéminente sur les marchés du monde entier. Ce résultat a été obtenu par les moyens que lo gouvernement fédéral se propose d'aclopter lui-même, suivant les circonstances. On croit que l'industrie laitière deviendra la bran. che de l'agriculture la plus lucrative dans notre pays.

\section{Le marché intérieur}

Dans le cours de l'amée dernière on a modifié le tarif, dans le but de protéger davantage le marché intérieur du cultirateur canadien. On a constaté par exemple que les producteurs améri. cains nous approvisionnaient de viandes, en grande partie. Dans le cours de l'année 1889, 3,795,105 livres de bœuf frais ont été expédiées au Canada par les abattoirs de Chicago et autres grandes villes, plus 27,147,491 livres de lard, de saindoux, de jambon et de bacon. On a cru avec raison que nos cultivateurs ne devaient pas être soumis à cette grave concurrence, autant que possible, et conformément à la politique de protection en vigueur, les droits sur ces articles ont été augmentés. En même temps les droits sur les fruits ont été rétablis. Nous pouvons dire maintenant que nos cultivateurs jouissent d'une grande protection, au point de vue surtout du marché local.

\section{Nouveaux marchés extérieurs}

Les efforts que fait le gouvernement pour obtenir de noureaux marchés sont d'une importance vitale pour la classe agricole. S'il réussit, les cultivateurs au Canada auront tout à y gagner. 